\title{
The Childhood Injury Prevention and Promotion of Safety Programme (CHIPPS)
}

\author{
Elizabeth Towner, Stephen Jarvis
}

\begin{abstract}
Aim
The CHIPPS Programme is based in the section of Community Child Health, Department of Child Health, University of Newcastle upon Tyne. The aim of this programme is to develop the scientific basis of child injury prevention and provide England with a centre of excellence in academic and applied research in the field. It sets out to develop a sound information base in the seven health districts of northen England (population 3 million) and to develop injury prevention programmes in the region. The intention is to develop methodologies that could be applied elsewhere. The main foci of the work are: children, unintentional injury, local and regional level work, and on the effects of social deprivation.
\end{abstract}

\section{Background \\ The Health of the Nation: A Strategy for Health in} England recognised the prevention of unintentional injury in childhood as one of its priority areas and set a target to reduce the death rate from accidents among children aged under 15 by at least $33 \%$ by 2005 . Key areas selected were major public health problems and those in which targets could be set and that were potentially preventable.

Unintentional injury is the leading cause of death in UK children betweeen 1 and 15 years of age. Deaths from this cause have a steeper social gradient than any other cause of death. In recent years these socioeconomic inequalities in childhood injury mortality have increased. For one of the most important types of injury, child pedestrian death, the UK has one of the poorest records in Europe.

In addition to being the main cause of death, unintentional injury is also a major cause of ill health in childhood. A study of childhood injuries in Newcastle in 1990 reported rates of 1.1 deaths, 166 hospital admissions, and 2149 accident and emergency attendances per 10000 resident children. The annual NHS cost of unintentional injuries in childhood in England and Wales has been estimated at $£ 200$ million per year. Other indirect costs include social and psychological costs to the children, their families, and society as a whole.

Comminty Child Health, Donald Court House, 13 Walker Terrace, Gateshead NE8 1EB, UK E Towner $\mathrm{S}$ Jarvis

Correspondence to: Dr Towner.
CHIPPS was established in June 1994 based on pilot projects in childhood injury prevention in the Department of Child Health that dated back to 1986. The Northern Region of
England has a long tradition of work in childhood injury prevention. The 'Thousand Families' study, which started in 1947, traced children's health and illness, including the accidents they had in their first 15 years of life. Dr Hugh Jackson was involved in this study and later continued his interest in childhood injury prevention in a series of studies on childhood poisoning and lacerations. $\mathrm{He}$ helped to organise the 'Children, the environment and accidents' conference in Newcastle in 1977 and stimulated interest among many of his local paediatric colleagues: Alan Craft, Jo Sibert, Geoffrey Lawson, and Allan Colver went on to make important contributions to the field in the 1970s and 1980 s. Hugh is still active as president of the Child Accident Prevention Trust and as a member of the CHIPPS team.

\section{Funding}

Funding for CHIPPS has been obtained from the Department of Health and the Northern and Yorkshire Regional Health Authority. This supports core posts in the programme. Research projects have been funded by the NHS Centre for Reviews and Dissemination, University of York; by the NHS R\&D Programme on Mother and Child Health; and by the Northern and Yorkshire Regional Health Authority.

\section{Main focus of research}

The main focus of the research in childhood injury prevention is around three themes: injury event data, injury risk data, and systematic reviews of the effects of preventive interventions.

\section{(A) INJURY EVENT DATA}

A severity coded register of accidental injury for children under the age of 16 years has been compiled for Gateshead residents. Three routine data sets are used to create the registerhospital inpatient data, coroner's inquest files, and police road traffic accident data. Medical and nursing records and questionnaires administered to parents/carers and children are used to document the nature, severity, circumstances, and management of all injuries leading to hospital admissions. An annual report is disseminated to community paediatricians, directors of public health, and other interested agencies such as the Gateshead Multi-Agency 
Prevention Group. It is hoped that the prototype developed in Gateshead will become a model for other districts in the region.

\section{(B) INJURY RISK DATA}

Self completed questionnaires have been developed to collect information from $11-14$ year old schoolchildren on exposure to injury risk and from the parents/carers of 1-3 year old children. Six thousand preschool and 18000 school questionnaires have been collected from Newcastle, Gateshead, Darlington and Teesdale, Northumberland, Cleveland, and Sunderland. The risk questionnaires for school children, for example, collect information on the number of roads crossed on journeys to and from school, the frequency of bicycling, the use of cycle helmets, where leisure time is spent, etc. Injury risk data provide good local information that can be used in the development and evaluation of prevention programmes. Risk data have also been used to monitor trends over time, of, for example, mode of travel to school or cycle helmet use.

\section{(C) SYSTEMATIC REVIEWS}

Reliable evidence about effectiveness of injury prevention is needed to apply effective measures more widely and to identify gaps in knowledge. A number of effectiveness reviews were conducted in pilot projects before the establishment of CHIPPS and then within the CHIPPS Programme. These have been conducted for the Health Education Authority, Northern and Yorkshire Regional Health Authority, the Dutch Centre for Health Promotion and Health Education, and the NHS Centre for Reviews and Dissemination, University of York. These reviews will be updated over the next four years. Emphasis has been placed both on the rigour of the evaluation and detailed accounts of the nature of the intervention.

\section{New research projects}

Recent new research projects that have been funded include:

(1) A randomised controlled trial of injury prevention in primary schools

(2) Effectiveness review update and methodological study

(3) A case-control study of scald injuries in preschool children

(4) A professional first aid training study

(1) The randomised controlled trial of an injury prevention programme, conducted in 15 primary schools, with 30 control schools includes a two year intervention targeting 7 and 9 year old pupils. The goal is to reduce school, sports and leisure injuries, reduce. hazards, and increase injury prevention aware-0 ness. Money has been allocated to fund environment modifications within the inter vention schools and to permit teachers to attend training courses. The evaluation wilg be conducted over a period of five years and will include the measurement of outcome? impact, and process. Outcome evaluation wilk include injury event data, both inpatient and outpatient, and $x$-ray data on injuries with 5 abbreviated injury scale (AIS) scores of two or greater. Impact evaluation will use injury riskw questionnaires for 7-9 year old children ancos their parents. Process evaluation will access the extent to which the programme has been implemented, assess the satisfaction of the school community with the programme, and document the nature of the intervention. This study has been funded by the NHS R\&D Programme in Mother and Child Health and began in October 1996.

(2) The effectiveness review study will sys $\overrightarrow{0}$ tematically assess the effectiveness of injury prevention interventions aimed at reducingo childhood (0-14 years) unintentional injurys and continue to update and disseminate thiso information for the use of practitioners, re $-\frac{0}{D}$ searchers, and policy makers. Updated reviews will be produced in 1998 and 2000, and $\overrightarrow{\overrightarrow{5}}$ review of the research methodologies in 1999. It is hoped that the database will be accessible on the Internet/World Wide Web in June 1998:

(3) The aim of the case-control study is to investigate the risk factors for severe scald injuries in early childhood ( $0-4$ years) in order to identify possible preventive strategies. The study will be conducted in seven district health authorities in the Northern Region of England and employs a case-control design with two controls for each case.

(4) The professional first aid training studyo is a pilot study to assess the level of knowledge and skills in first aid among three groups of professionals: health visitors, school nurses, and nursery nurses. We hope to identify气 training needs and apply this information in the development of training packages. The study will consist of postal questionnaires and in-depth interviews and will be conducted in three districts in the Northern Region of England.

Further projects are planned to explore ad variety of alternative injury event case definitions for epidemiological and outcome studies. Similar methodological investigations are being developed to extend the range and accuracy of population wide prevalence measures. 